\title{
Relationship between the Presence of the nalC Mutation and Multidrug Resistance in Pseudomonas aeruginosa
}

\author{
Nourkhoda Sadeghifard, ${ }^{1}$ Azar Valizadeh, ${ }^{2}$ Mohammad Reza Zolfaghary, ${ }^{2}$ \\ Mohammad Hossien Maleki, ${ }^{1}$ Abbas Maleki, ${ }^{1}$ Reza Mohebi, ${ }^{1}$ Sobhan Ghafourian, ${ }^{1}$ \\ and Afra Khosravi ${ }^{1}$ \\ ${ }^{1}$ Clinical Microbiology Research Center, Ilam University of Medical Sciences, Ilam 693917134, Iran \\ ${ }^{2}$ Department of Microbiology, Qom Branch, Islamic Azad University of Qom, Qom, Iran \\ Correspondence should be addressed to Sobhan Ghafourian, sobhan.ghafurian@gmail.com
}

Received 10 January 2012; Revised 17 February 2012; Accepted 17 February 2012

Academic Editor: Todd R. Callaway

Copyright (C) 2012 Nourkhoda Sadeghifard et al. This is an open access article distributed under the Creative Commons Attribution License, which permits unrestricted use, distribution, and reproduction in any medium, provided the original work is properly cited.

Objectives. The current study was conducted to determine the relationship between the presences of significant multidrug resistance in Pseudomonas aeruginosa (P. aeruginosa) having intact mexR genes (nalC) to different antibiotics. Methods. In order to identify nalC, fifty strains of $P$. aeruginosa were obtained. All isolates were found in urinary tract infections. They were evaluated against different antibiotics. The nalC mutant was identified by PCR. Results. The 50 clinical isolates of $P$. aeruginosa originated from two hospitals in Iran, in which 32 isolates were found in Milad hospital, and 18 isolates were collected in the Ilam Hospital. The results in Milad hospital of nalC revealed that all $P$. aeruginosa resistant to oxacillin showed the presence of nalC. In Ilam hospital only three $(16.6 \%)$ isolates were resistant to oxacilin and aztreonam, and among these three isolates only one isolate revealed resistance to ceftazidime and amikacin. The resistant isolates showed the presence of both OXA-10 and nalC. Conclusion. Our results showed that the presence of nalC was observed among $P$. aeruginosa resistance to oxacilin. Thus, the finding suggested relationship between oxacilin resistance and presence of nalC and consequently overproduction of the MexABOprM efflux system.

\section{Introduction}

$P$. aeruginosa is an opportunistic human pathogen characterized by an innate resistance to multiple antimicrobials [1]. $P$. aeruginosa is responsible for $10-15 \%$ of the nosocomial infections worldwide [2]. Often these infections are hard to treat due to the natural resistance of the species as well as to its remarkable ability of acquiring further mechanisms of resistance to multiple groups of antimicrobial agents.

Oxacillinases (OXA-type enzymes) belong to molecular class D and functional group 2 [3]. Classical OXA enzymes (OXA-1, OXA-2, and OXA-10) determine resistance to carboxypenicillins and ureidopenicillins but not to ceftazidime [4]. Resistance to ticarcillin and piperacillin resulting from production of OXA-2 enzymes is lower than the resistance that develops when OXA-10 and OXA-1 oxacillinases are produced [4]. Generally, P. aeruginosa clinical isolates are less susceptible than Enterobacteriaceae to most classes of antimicrobials. For a long time the main reason for this natural resistance was considered to be the low outer membrane permeability due to the presence of proteins with high molecular mass about $50 \mathrm{kDa}[5]$.

Efflux contributes to the development of multiple resistances to all strategic antipseudomonal antibiotics and is mediated by four genetically different three component efflux systems that belong to the resistance-nodulation-division (RND) family: MexA-MexB-OprM, MexC-MexD-OprJ, MexE-MexF-OprN, and MexX-MexY-OprM. The first component is a protein located in the cytoplasmic membrane (MexB, MexD, MexF, and MexY) that operates as an energydependent pump with wide substrate specificity. The second component is a gated outer membrane protein (OprM, OprJ, OprN, and OprM). The third protein (MexA, MexC, MexE, and MexX) is located in the periplasmic space and links the other two [6].

There are also other mutants called nalC that have intact mexR genes [7]. nalC mutants originate from the wild-type $P$. aeruginosa $\mathrm{PAO} 1$ and are characterized by a mutation in 
the PA3721 gene [8]. The protein encoded by this gene is a repressor of a two-gene operon; its function is unclear, and its overexpression in nalC mutants leads to overproduction of the MexAB-OprM efflux system.

The current study was conducted to determine the relationship between the presence of the nalC mutation in $P$. aeruginosa and its resistance to antibiotics.

\section{Material and Methods}

2.1. Bacterial Strains. In order to identify nalC, fifty strains of Pseudomonas aeruginosa obtained during the period of March 2010 to August 2010 in the Milad and Ilam hospitals, from which 32 isolates were from the Milad hospital, and 18 isolates were from the Ilam hospital. All isolates were isolated from urinary tract infections.

$P$. aeruginosa which was resistant to cefotaxime, ceftazidime, amikacin, oxacillin, aztreonam, and ticarcillin was ESBLs positive, and the presence of OXA- 2 and OXA- 10 was assayed by the PCR for detection of ESBLs genes.

2.2. PCR. PCR was performed for detection of OXA-10 and OXA-2 genes. The primers used were as follows:

OXA-2 gene; Forward 5-GCC AAA GGC ACG ATA GTT GT-3;

Reverse 5-GCG TCC GAG TTG ACT GCC GG-3;

OXA-10 gene; Forward: 5-GT CTT TCG AGT ACG GCA TTA-3;

Reverse 5-ATT TTC TTA GCG GCA ACT TAC-3.

2.3. PCR for Detection of NalC. NalC was identified by using of Forward: 5-TCA ACC CTA ACG AGA AAC GCT-3 and Reverse 5-TCC ACC TCA CCG AAC TGC-3.

\section{Results}

The 50 clinical isolates of $P$. aeruginosa originated from two hospitals in Iran, which from 32 isolates were collected in the Milad hospital, and 18 isolates collected in the Ilam Hospital. After the identification of isolates as P. aeruginosa, they were evaluated for susceptibility to different antibiotics. The finding in Milad hospital revealed that $87.1 \%(n=25)$ of the isolates that expressed resistance to oxacilin and resistance to cefotaxime, ceftazidime, amikacin, aztreonam, and Ticarcillin were $62.5 \%(n=20), 50 \%(n=16), 56.25 \%(n=$ $18)$, and $68.75 \%(n=22)$, respectively. The findings showed that all oxacilin resistant strains harbored OXA-10, while only 2 isolates showed OXA-2 genes. Therefore, two isolates harbored both OXA-10 and OXA-2. The results of determining nalC revealed that all P.aeruginosa resistant to oxacilin showed the presence of nalC. Therefore, $87.1 \%$ of $P$. aeruginosa harbored nalC, in addition, 7 P. aeruginosa which were negative for nalC also were susceptible to the others antibiotics. There was a significant difference observed between the presence of nalC and oxacilin resistant strains. In Ilam hospital only three (16.6\%) isolates were resistant to oxacilin and aztreonam, and among these three isolates only one isolate was resistant to ceftazidime and amikacin. The resistant isolates showed the presence of both OXA-10 and nalC. The findings showed that no nalC was observed in sensitive isolates in both hospitals. A significant difference was observed among resistant strains to oxacilin and the presence of nalC, while no significant difference occurred among resistance strains to the other antibiotics and nalC.

\section{Discussion}

P. aeruginosa presents a therapeutic challenge for treatment of both community-acquired and nosocomial infections. Unfortunately, selection of the most appropriate antibiotic is complicated by the ability of $P$. aeruginosa to develop resistance to multiple classes of antibacterial agents, even during the course of treating an infection [9]. Epidemiological outcome studies have shown that infections caused by drugresistant $P$. aeruginosa are associated with significant increases in morbidity, mortality, the need for surgical intervention, the length of hospital stay and chronic care, and the overall cost of treating the infection [10].

Resistance to ticarcillin and piperacillin resulting from the production of OXA-2 enzymes is lower than the resistance that develops when OXA-10 and OXA-1 oxacillinases are produced [4]. The results of this study showed that OXA2 enzyme was found in two isolates which demonstrated resistance to ticarcillin.

The results showed a significant difference between nalC and oxacilin resistant strains.

MexA-MexB-OprM overproduction often occurs in clinical isolates of $P$. aeruginosa, and usually it is a result of increased transcription of the mexA-mexB-oprM operon due to mutations in the chromosomal gene encoding the MexR repressor protein, that is, mutations at the mexR locus [11]. The protein encoded by this gene is a repressor of a two-gene operon; its function is unclear, and its overexpression in nalC mutants leads to overproduction of the MexAB-OprM efflux system.

In conclusion, Nalc exists as an important factor in the increased expression of pumps MEXAB-OPRM, started its presence in samples containing ESBL has been reported in the world. Several reasons can explain the relationship between nalc and beta-lactamase such as the effects of creation of nalc, is the increase of expression of porin MEX ABOPRM. Our results showed that the presence of nalC occurred concomitant with resistance to oxacilin. Thus, the finding suggested a relationship between oxacilin resistance and the presence of nalC and consequently overproduction of the MexAB-OprM efflux system. Our results revealed that more study is needed for finding a way which is able to reduce the oxacilin resistance and lead to reduction of production of MexAB-OprM efflux system.

\section{References}

[1] R. E. W. Hancock and D. P. Speert, "Antibiotic resistance in Pseudomonas aeruginosa: mechanisms and impact on 
treatment," Drug Resistance Updates, vol. 3, no. 4, pp. 247-255, 2000.

[2] D. S. Blanc, C. Petignat, B. Janin, J. Bille, and P. Francioli, "Frequency and molecular diversity of Pseudomonas aeruginosa upon admission and during hospitalization: a prospective epidemiologic study," Clinical Microbiology and Infection, vol. 4, no. 5, pp. 242-247, 1998.

[3] K. Bush, G. A. Jacoby, and A. A. Medeiros, "A functional classification scheme for $\beta$-lactamases and its correlation with molecular structure," Antimicrobial Agents and Chemotherapy, vol. 39, no. 6, pp. 1211-1233, 1995.

[4] F. Bert, Z. Ould-Hocine, M. Juvin et al., "Evaluation of the Osiris expert system for identification of $\beta$-lactam phenotypes in isolates of Pseudomonas aeruginosa," Journal of Clinical Microbiology, vol. 41, no. 8, pp. 3712-3718, 2003.

[5] D. M. Livermore, "Penicillin-binding proteins, porins and outer-membrane permeability of carbenicillin-resistant and -susceptible strains of Pseudomonas aeruginosa," Journal of Medical Microbiology, vol. 18, no. 2, pp. 261-270, 1984.

[6] D. M. Livermore, "Multiple mechanisms of antimicrobial resistance in Pseudomonas aeruginosa: our worst nightmare?" Clinical Infectious Diseases, vol. 34, no. 5, pp. 634-640, 2002.

[7] R. Srikumar, C. J. Paul, and K. Poole, "Influence of mutations in the mexR repressor gene on expression of the MexA-MexBOprM multidrug efflux system of Pseudomonas aeruginosa," Journal of Bacteriology, vol. 182, no. 5, pp. 1410-1414, 2000.

[8] L. Cao, R. Srikumar, and K. Poole, "MexAB-OprM hyperexpression in NalC-type multidrug-resistant Pseudomonas aeruginosa: identification and characterization of the nalC gene encoding a repressor of PA3720-PA3719," Molecular Microbiology, vol. 53, no. 5, pp. 1423-1436, 2004.

[9] J. Bisbe, J. M. Gatell, J. Puig et al., "Pseudomonas aeruginosa bacteremia: univariate and multivariate analyses of factors influencing the prognosis in 133 episodes," Reviews of Infectious Diseases, vol. 10, no. 3, pp. 629-635, 1988.

[10] V. Aloush, S. Navon-Venezia, Y. Seigman-Igra, S. Cabili, and Y. Carmeli, "Multidrug-resistant Pseudomonas aeruginosa: risk factors and clinical impact," Antimicrobial Agents and Chemotherapy, vol. 50, no. 1, pp. 43-48, 2006.

[11] K. Saito, H. Yoneyama, and T. Nakae, "NalB-type mutations causing the overexpression of the MexA-MexB-OprM efflux pump are located in the mexR gene of the Pseudomonas aeruginosa chromosome," FEMS Microbiology Letters, vol. 179, no. 1, pp. 67-72, 1999. 



Submit your manuscripts at

http://www.hindawi.com
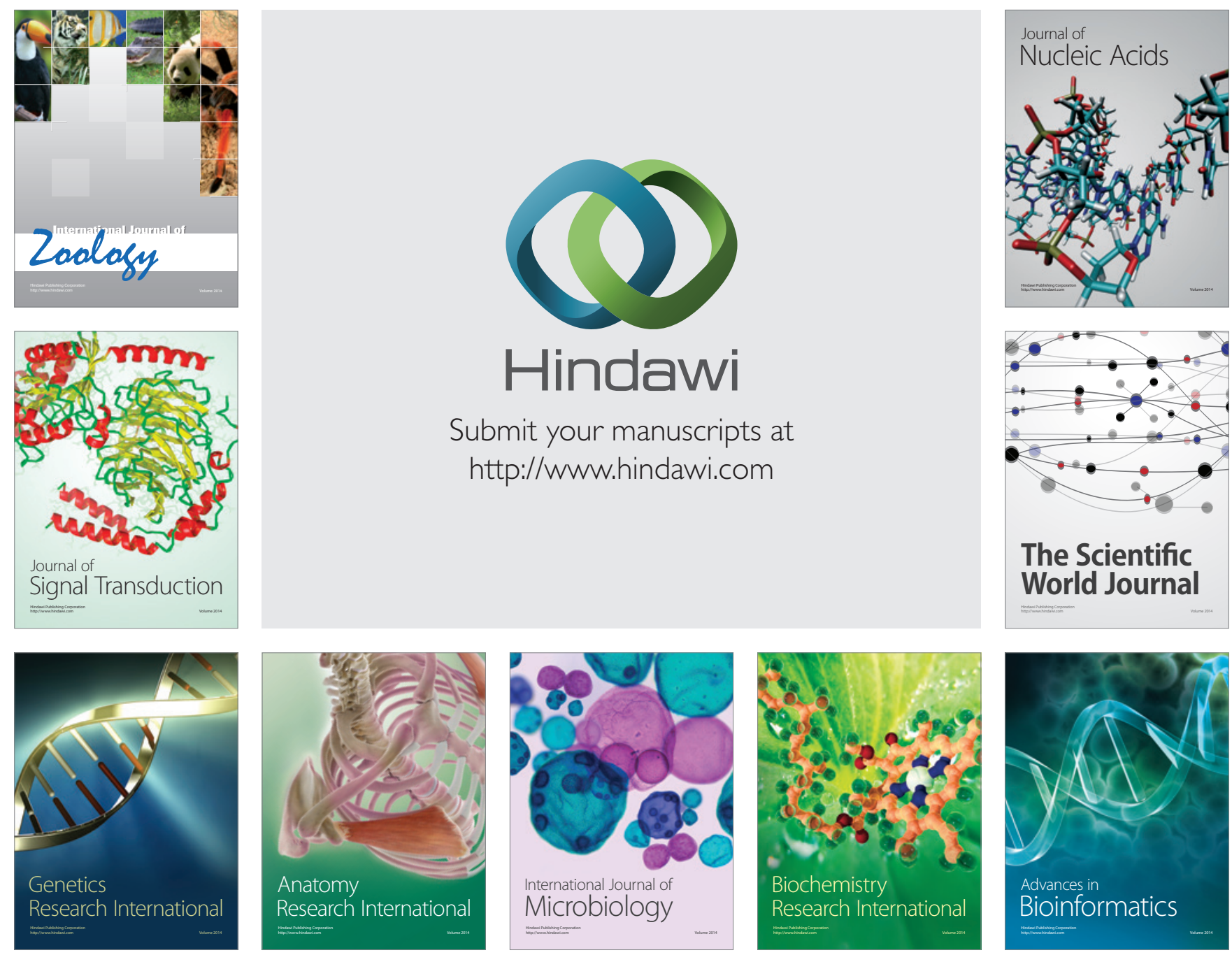

The Scientific World Journal
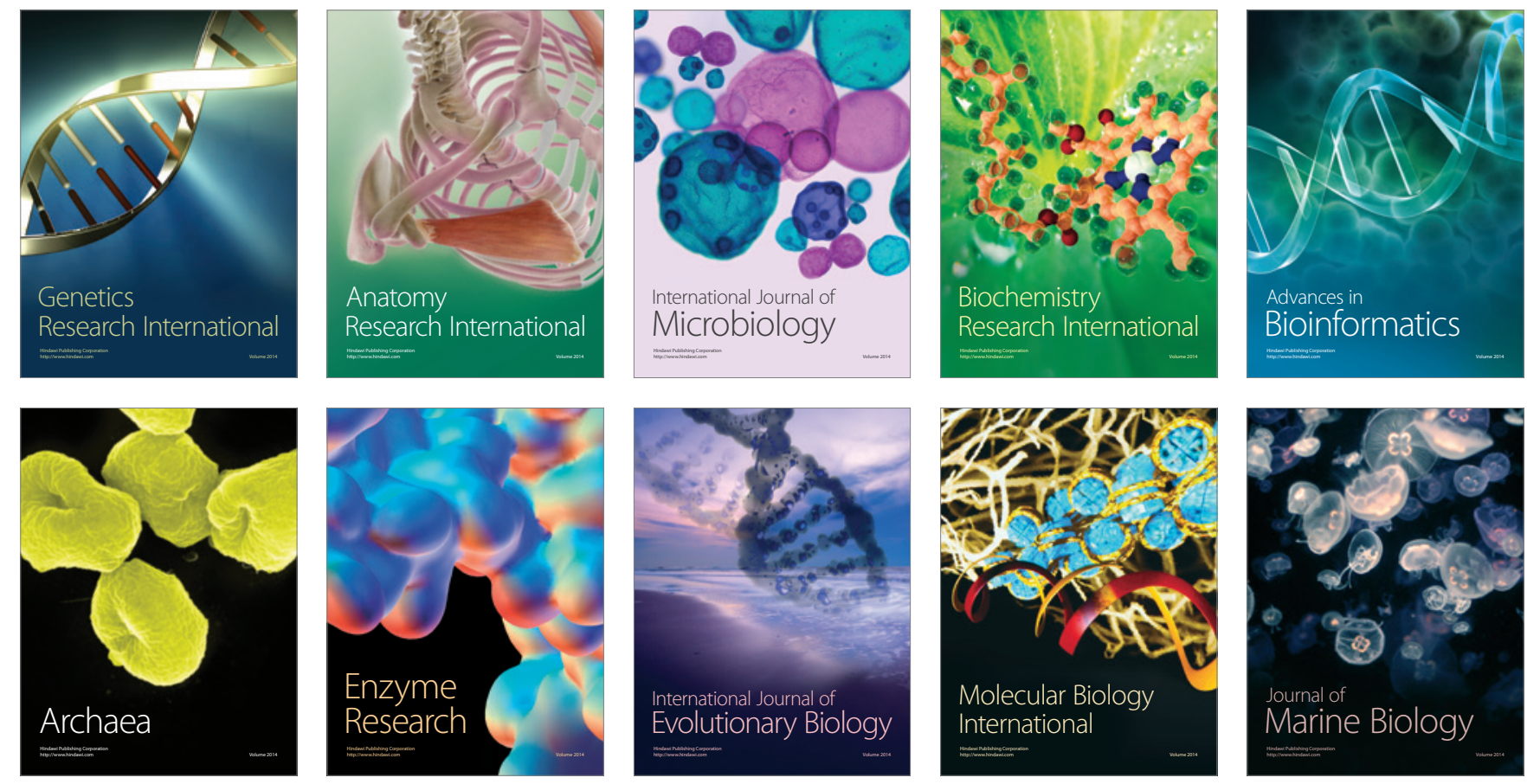